\title{
THE EFFECT OF TRAPPED BACKSTREAMING IONS ON BEAM FOCUS AND EMITTANCE IN RADIOGRAPHIC ACCELERATORS
}

\author{
T. Houck, J. McCarrick, LLNL, Livermore, CA
}

\section{Abstract}

The use of electron beam accelerators in advanced X-ray radiography requires that the beam be focused to a very small spot size on a metallic bremsstrahlung converter target. The energy deposition of the beam into a small volume of target material rapidly converts the target into a high-density plasma. The space-charge density of the focused beam sets up a strong electric field at the surface of the grounded target, which then accelerates ions of target plasma and lighter contaminants into the beam. These "backstreaming ions" form a long channel which partially neutralize the space charge of the beam, disrupting the beam focus and degrading the radiographic performance. One solution to this problem is to confine the backstreaming ions in a short channel with a selfbiased inductive trap. Such a trap has been proposed for testing on the ETA-II accelerator. We will present numerical simulations which show the effect of the high trapped-ion charge density on the beam spot size and emittance.

\section{INTRODUCTION}

Accelerator systems for X-ray radiography transport a high-current relativistic electron beam to a final-focus system, where the beam is concentrated to a small $(\sim 1$ $\mathrm{mm})$ spot size on a metallic converter target. X-rays are produced via bremsstrahlung in the converter material. The high energy deposition of the beam into the target rapidly heats it, creating a plasma of target material and any light contaminants. Since the metallic target and subsequent plasma form an equipotential surface, and since the target system is usually grounded, the high space-charge density of the electron beam generates a large axial electric field in front of the target. This electric field extracts ions from the target plasma and quickly accelerates them upstream into the beam, in a process referred to as "ion backstreaming." The ion space charge neutralizes the beam space charge, so that the beam's own magnetic field overfocuses the beam and degrades the X-ray production. This process is shown schematically in the upper portion of Figure 1. Simple analytic models of this process [1] show that a very small supply of ions, parts per billion relative to the solid density of the target, is enough to generate space-charge limited (SCL) ion flow into the beam, on a fairly fast time scale (10-20ns) relative to a typical beam pulse (50-60ns) if light ions are present.

The best tactic for avoiding backstreaming ions would be to suppress completely the formation of plasma at the target, but no plausible scheme for accomplishing this has been found. Another technique is to dynamically adjust the

beam and its focusing system to compensate for the presence of the ions [1]. However, the most straightforward technique is to inductively [2] or resistively [3] bias the target to counteract the axial electric field produced by the electron beam.

In the inductive case, a modified accelerating cell is placed in front of the converter target and operated in reverse of its normal polarity, creating a potential well in front of the target which traps ions before they can form a channel of sufficient length (the disruption length) to defocus the beam. This is shown in the lower portion of Figure 1. Note that during the flattop of the voltage pulse on the induction cell, a static field model can be used and the concept of a potential is applicable.
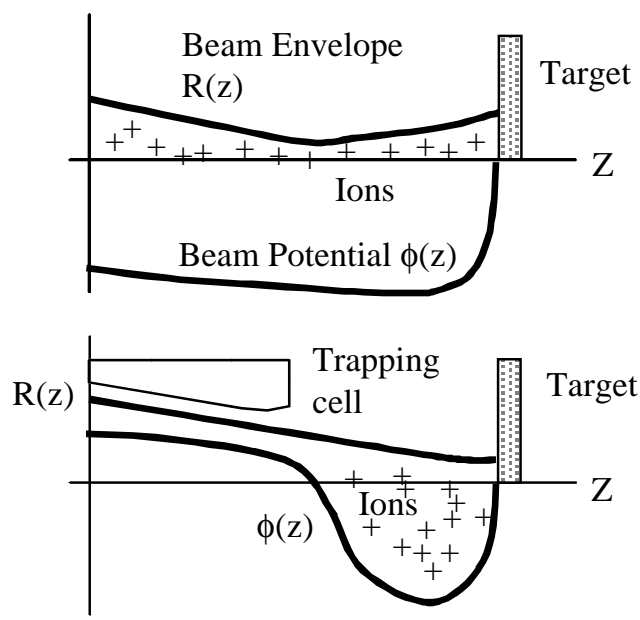

Figure 1. Untrapped (upper) and trapped (lower) backstreaming ion configurations.

Such a cell has been designed and will be tested as part of the ongoing experimental campaign on the ETA-II accelerator (see, for example, [4]) to evaluate this technology for use in the DARHT-II project. Previous modeling [2] of this cell shows that it can confine backstreaming ions, but these simulations did not include the self-consistent behavior of the electron beam as modified by the trapped ions. Since the results showed that the trapped ion density is much higher than the SCL value normally used to estimate the disruption length, it is not clear that trapping the ions actually preserves the beam spot size; it is also possible that such a dense ion channel produces emittance growth, which degrades X-ray production. Therefore, we have performed fully self- 
consistent numerical modeling of this inductive ion trap using DARHT-II beam parameters.

\section{NUMERICAL RESULTS}

Two types of simulations have been performed of the inductive ion trap. Time-dependent particle-in-cell (PIC) modeling has been done, and when it was observed that the system settles into a steady-flow configuration, a Vlasov electron model was employed for more accurate calculation of the beam envelope and emittance.

The geometry and typical beam and ion configuration, as used in the PIC runs, are shown in Figure 2. The simulation runs for 20ns; hydrogen ions are emitted in SCL flow beginning at $1 \mathrm{~ns}$, after the beam has ramped up to a steady current of $2 \mathrm{kA}$ and an energy of $20 \mathrm{MeV}$. The beam hard-edge radius at the target, prior to ion emission, is slightly over $0.5 \mathrm{~mm}$. The voltage across the gap of the induction cell is $400 \mathrm{kV}$.



Figure 2. Computational geometry, showing beam, ions, aperture plate, and induction cell electrode.

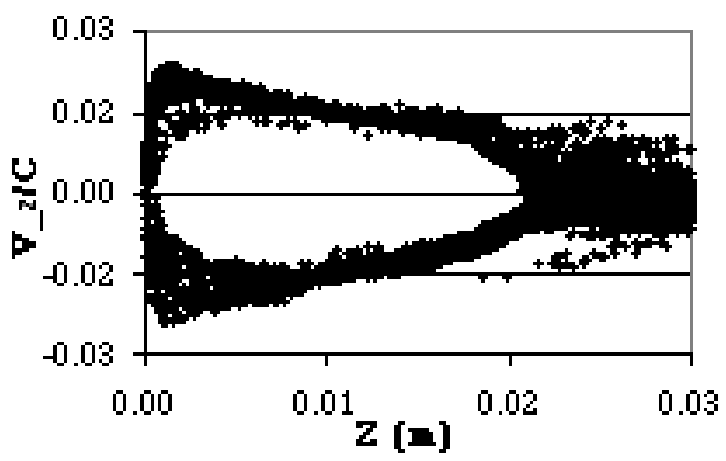

Figure 3. Steady circulation in ion Z-Vz phase space.

Figure 3 shows the $z-v_{z}$ phase space of the ions at 20ns. The ions have reached a steady circulation in the trapping field, suppressing further emission from the target plasma. (Note that over the duration of a beam pulse, the target plasma does not expand significantly from the original surface.) Figure 4 shows the ratio of ion charge density to beam charge density on the axis; they are of the same order, as opposed to the $\sim 10 \%$ value reached in unconfined SCL flow. The ion channel length is quite short, however, at about $3.4 \mathrm{~cm}$. Figure 5 shows the beam spot size and emittance at the target as a function of time. Here we see that the beam spot size is controlled, but at the expense of substantial emittance growth.

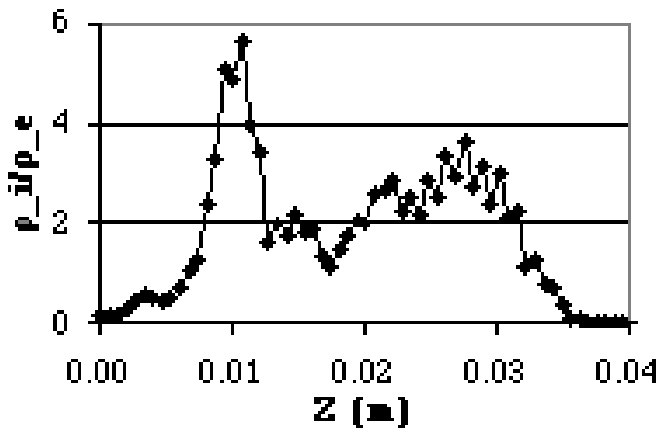

Figure 4. On-axis ratio of ion charge to beam charge.

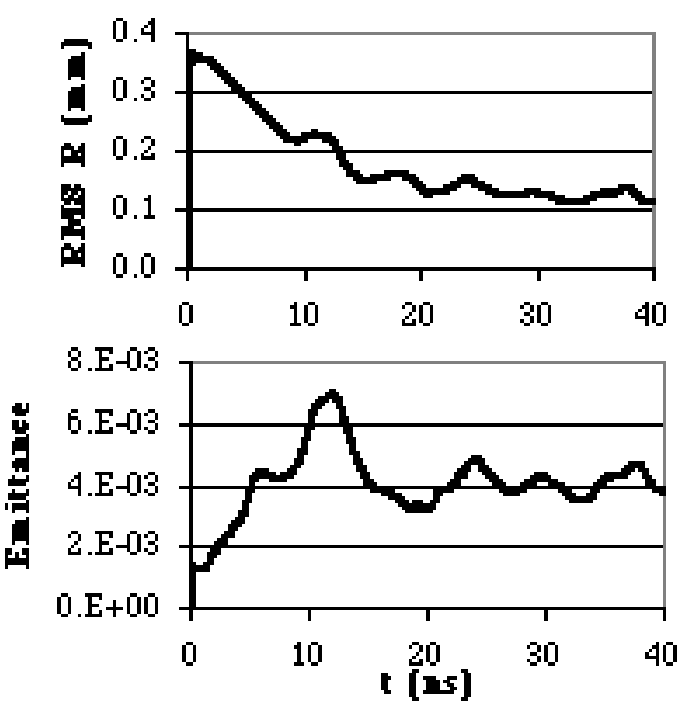

Figure 5. Beam envelope and emittance, at the target plane, as a function of time.

Because of concerns that the PIC code generates significant numerical emittance growth, the steady-state ion density was fed into a Vlasov electron model to confirm the degradation of the beam emittance. The beam envelope and emittance as a function of axial distance from the target are shown in Figure 6. It is important to note that the target plane is at $\mathrm{z}=0.3 \mathrm{~cm}$ in this model. These results show less emittance growth than in the PIC runs but nevertheless a substantial rise over the initial value of $1200 \pi$-mm-mrad (normalized).

Parametric studies have been performed to examine the variation of spot size control and emittance growth with cell voltage and gap size. However, due to strong engineering constraints on combinations of voltage and gap size that will avoid breakdown in the presence of an expanding plasma, and the limit on channel length to prevent spot size variation, no significant departure from the behavior shown here could be attained. 


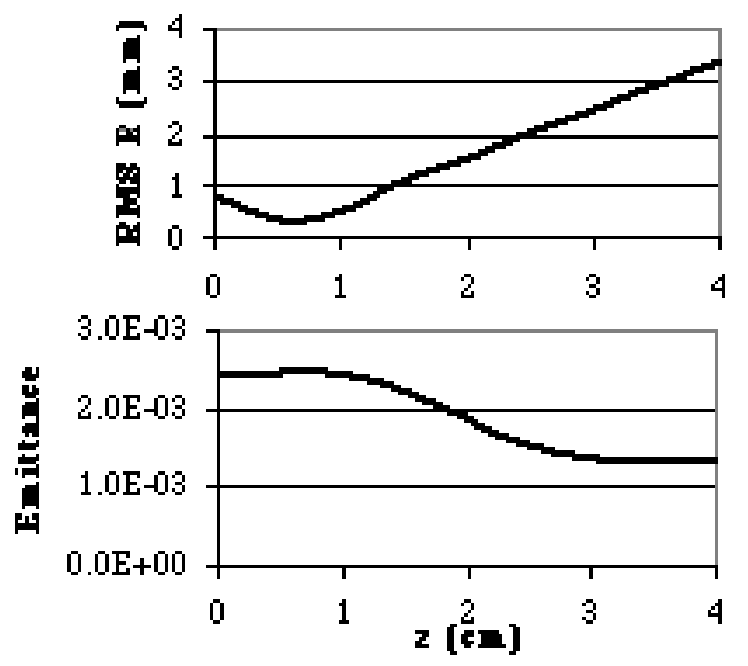

Figure 6. Steady state spatial profile of beam envelope and emittance from Vlasov model. Note the target plane is at $\mathrm{z}=0.3 \mathrm{~cm}$ in these results.

\section{CONCLUSIONS}

A fully self-consistent model of an electron beam and backstreaming ions in an inductive trap has been studied, to see the effect of the high trapped ion charge density on the beam. The beam spot size is controlled successfully as the ions are confined to a short channel, and reach a steady-state configuration. However, there is considerable emittance growth in the beam due to nonlinear focusing. Parametric studies performed within the engineering constraints of an induction cell have not yielded an alternate design.

\section{ACKNOWLEDGEMENTS}

We would like to thank Peter Rambo for insightful discussions and code benchmarking. This work performed under the auspices of the U.S. Department of Energy by LLNL under contract W-7405-ENG-48.

\section{REFERENCES}

[1] Y-J. Chen, et. al., "Controlling Backstreaming Ions from X-ray Converter Targets with Time Varying Final Focusing Solenoidal Lens and Beam Energy Variation." Proceedings, 19th International Linear Accelerator Conference, Chicago, IL, 1998

[2] J. McCarrick, et. al., "Trapping Backstreaming Ions from an X-ray Converter Using an Inductive Cell." Proceedings, 19th International Linear Accelerator Conference, Chicago, IL, 1998.

[3] T. Kwan, et. al., "Design Simulation for Spot Size Stabilization in ITS/DARHT."' Proceedings, 19th International Linear Accelerator Conference, Chicago, IL, 1998

[4] G. A. Westenskow, et. al., "Experimental Investigation of Beam Optics Issues at the Bremsstrahlung Converters for Radiographic Applications." Proceedings, 19th International Linear Accelerator Conference, Chicago, IL, 1998 